\title{
La estructura genética de la ostra chilena (Ostrea chilensis Philippi, 1845) en poblaciones naturales del sur de Chile, basada en análisis con marcadores RAPDs
}

\author{
The genetic structure of the Chilean oyster (Ostrea chilensis Philippi, 1845) in natural populations of southern \\ Chile based on RAPDs analysis \\ Jorge E. Toro ${ }^{1}$ y Carolina P. González ${ }^{1}$ \\ ${ }^{1}$ Instituto de Biología Marina 'Dr. Jürgen Winter', Universidad Austral de Chile, Casilla 567, Valdivia, Chile \\ jtoro@uach.cl
}

\begin{abstract}
Natural populations of Ostrea chilensis have suffered strong declines during the last two decades due to overfishing that forced in some locations to carry out restocking and culture with juvenile oysters from other local populations. It has been proposed that this type of intervention may have affected the levels of genetic variation among natural populations. Here we analyzed the genetic variation in two natural populations and in three populations affected by restocking of $O$. chilensis, sampled along its whole natural distribution, by means of Random Amplified Polymorphic DNA (RAPD) analyses. The values of Nei's genetic distance did not reveal significant differences among populations (0.0713 and 0.1549). The Mantel test using 5000 randomizations, showed a significant correlation between genetic and geographic distance. Melinka, the most geographically distant population, was the most genetically differentiated. The levels of gene flow $(\mathrm{Nm}=1.587)$ are strong enough to prevent differentiation due to genetic drift. With these results, the genetic-population structure of $O$. chilensis, can be characterized a panmictic population, in which, the level of gene flow prevents significant genetic differentiations among populations. However, the populationgenetic structure of this species does not depend exclusively on its breeding strategy, since other factors such as: oceanographic and human mediated gene flow probably exert a large influence to the genetic structure of the studied populations.
\end{abstract}

Key words: Gene flow, oysters, genetic variation

\section{Introducción}

Ostrea chilensis (Philippi, 1845) es un recurso marino de gran importancia comercial en Chile. Los desembarques provenientes de bancos naturales de esta especie entre 19381942 fueron en promedio de 1000 toneladas anuales, provocando una sobreexplotación de éstos y como consecuencia una brusca baja en el desembarco (Lépez 1983). A partir de 1954, el estado de Chile efectuó acciones
Resumen.- Las poblaciones naturales de Ostrea chilensis han sufrido fuertes disminuciones debido a la sobrepesca, lo cual ha forzado en algunos sectores a llevar a cabo repoblamiento y cultivo con ostras juveniles provenientes de otras localidades. Se ha planteado que este tipo de intervención podría haber afectado los niveles de variación genética en las poblaciones naturales. Se analizó la variabilidad genética, a través del análisis con ADN Polimórfico Amplificado Aleatoriamente (RAPD), en dos poblaciones naturales $\mathrm{y}$ tres poblaciones afectadas por el repoblamiento de $O$. chilensis, muestreadas a lo largo de su distribución natural. Los valores de distancia genética de Nei no revelaron diferencias significativas entre poblaciones $(0,0713-0,1549)$. La prueba de Mantel con 5000 permutaciones evidenció una correlación significativa entre la distancia geográfica y la genética. La población de Melinka, situada a mayor distancia geográfica mostró la mayor diferenciación genética. Los niveles de flujo génico $(\mathrm{Nm}=1,587)$ son suficientemente fuertes para impedir diferencias genéticas en las poblaciones debido a la deriva génica. Con estos resultados se caracterizó la estructura genético-poblacional de $O$. chilensis como una población panmíctica, en la cual el flujo génico no permite una significativa diferenciación genética interpoblacional. Sin embargo, la estructura genéticopoblacional de esta especie no depende exclusivamente de su estrategia reproductiva, sino que existen otros factores oceanográficos y antropogénicos, que probablemente estarían influenciando la conformación genética de las poblaciones estudiadas.

Palabras clave: Flujo génico, ostras, variabilidad genética para regularizar la extracción de este recurso, imponiendo una veda y un tamaño mínimo de extracción, pero fue solo a fines de la década de 1960, con el inicio del cultivo de la ostra, que el desembarque de esta especie se incrementó nuevamente. En 1993 el desembarque de la ostra chilena alcanzó las 698 t sin embargo, entre 1994 y 2004 los desembarques se mantuvieron muy por debajo de esta cifra (SERNAPESCA 2004). Durante el año 2007 el desembarque total de O. chilensis fue de $191 \mathrm{t}$, de las cuales $187 \mathrm{t}$ 
correspondieron a la producción de centros de cultivo.

Las poblaciones naturales de esta especie han sufrido fuertes disminuciones debido a la sobrepesca, lo que indujo en algunos sectores a un repoblamiento con ostras de otras poblaciones locales (Toro \& Chaparro 1990, Bustos et al. 1991) y al traslado de juveniles desde la población natural

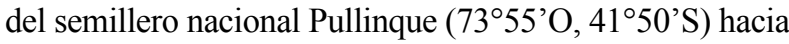
los distintos centros de cultivo en el sur de Chile. Se ha planteado que estas intervenciones podrían haber afectado los niveles de variación genética en las poblaciones naturales, reduciendo su diferenciación genética inter-poblacional, principalmente de las poblaciones situadas en la parte norte de la isla de Chiloé (Toro \& Aguila 1996). Por otro lado, la biología reproductiva de esta especie se caracteriza por poseer un desarrollo larval al interior de la cavidad paleal de la hembra (Ramorino 1970), lo cual impide una alta dispersión larval (Toro \& Chaparro 1990). La incubación larval se prolonga por aproximadamente siete semanas, presentando variaciones atribuidas a condiciones ambientales del cuerpo de agua imperantes durante la época reproductiva (Winter et al. 1984, Toro \& Morandé 1998). La larva pedivelígera una vez eclosionada, posee un período de vida planctónica reducido, que va desde 5 min a 24 h (Di Salvo \& Martínez 1985, Toro \& Chaparro 1990, Toro et al. 1995).

Debido a la sobreexplotación de los bancos naturales, a los procesos de traslado de juveniles y semillas y a la poca capacidad de dispersión a nivel larval de esta especie es que es de gran interés analizar el estado de la estructura genéticopoblacional y entender los mecanismos de diferenciación entre poblaciones a través de su rango de distribución geográfica en el sur de Chile (Scheltema 1989, Toro \& Aguila 1996, Silva \& Russo 2000, Thorpe et al. 2000). Del mismo modo, el estudio de los patrones de subdivisión poblacional y la magnitud del flujo génico entre poblaciones de esta especie son importantes para entender los procesos microevolutivos que les afectan (Slatkin \& Barton 1989), y generar políticas de manejo para su conservación.

Dentro de los numerosos marcadores moleculares utilizados en genética poblacional, se destaca la técnica de ADN Polimórfico Amplificado Aleatoriamente (Random Amplified Polymorphic DNA, RAPD), desarrollada por Williams et al. (1990) y Welsh \& McClelland (1990). El método consiste en amplificar segmentos de ADN al azar mediante PCR ('Polimerase Chain Reaction'), usando como partidores ('primers') secuencias arbitrarias y cortas de ADN de alrededor de 10 nucleótidos, para luego comparar los segmentos amplificados en un gel de agarosa (Williams et al. 1990, Welsh \& McClellannd 1990). Los RAPDs han sido utilizados para construir mapas genéticos de una gran variedad de especies, como también para la diferenciación de procariotas, plantas (Aagaard et al. 1998) y peces (Hassanien et al. 2004), así como en trabajos enfocados a la conservación (Pearson et al. 2002, Callejas \& Ochando 2002). En especies marinas de importancia comercial, su uso ha ido en incremento en los últimos años (Patwary et al. 1995, Elo et al. 1997, Star et al. 2003, Hermosilla 2004, Toro et al. 2004, Keyvanshokooh \& Kalbassi 2006). Algunas de las ventajas de los RAPDs tienen que ver con su bajo costo, mayor rapidez en la generación de datos y el menor requerimiento de muestra de tejido, al compararlo con otras técnicas moleculares (Caetano-Anolles et al. 1991a,b, Lynch \& Milligan 1994, Bardakci \& Skibinski 1994, Silva \& Russo 2000). El uso de la técnica RAPDs requiere de una verificación en la repetibilidad de sus resultados, en especial debido a factores tales como la calidad y concentración del ADN, el tipo de polimerasa, la concentración de magnesio, el tipo de termociclador y el programa utilizado para la amplificación (Liu \& Cordes 2004). Sin embargo, una vez estandarizada la técnica se logra una alta repetibilidad que permite ser utilizada en estudios de sistemática y ecología (Patwary et al. 1994, Holms et al. 2003, Star et al. 2003, Toro et al. 2004).

El presente estudio buscó caracterizar genéticamente las poblaciones naturales de Ostrea chilensis en el sur de Chile y evaluar el grado de variabilidad genética entre ellas, otorgando datos importantes para la conservación y el manejo de este recurso.

\section{Material y métodos}

\section{Muestreo de poblaciones}

Se recolectaron ejemplares de Ostrea chilensis procedentes de cinco poblaciones naturales, situadas a lo largo de la costa interior de Chiloé e Islas Guaitecas (Fig. 1). Dos de éstas corresponden a poblaciones naturales sin los efectos de repoblamiento, Pullinque (41 ${ }^{\circ} 51^{\prime}$ 'S; $73^{\circ} 58^{\prime} \mathrm{W}$ ) y Melinka

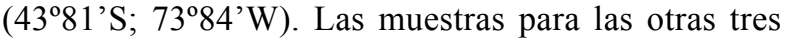
poblaciones se recolectaron en las localidades de

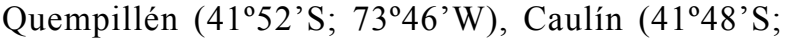

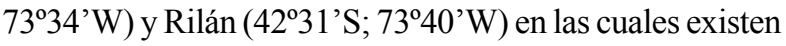
efectos de repoblamiento a través de transferencia de juveniles para actividades de cultivo. Estas localidades fueron seleccionadas porque abarcan todo el rango actual de distribución natural de la especie en el sur de Chile. Los muestreos fueron realizados en el submareal mediante buceo autónomo y de cada localidad se recolectó un mínimo de 60 individuos adultos elegidos al azar (36,9-65,1 mm). De cada ejemplar se extrajo un trozo de tejido del borde del manto, el cual se fijó en etanol al 95\% en tubos Eppendorf los que se mantuvieron posteriormente a $-20^{\circ} \mathrm{C}$. 

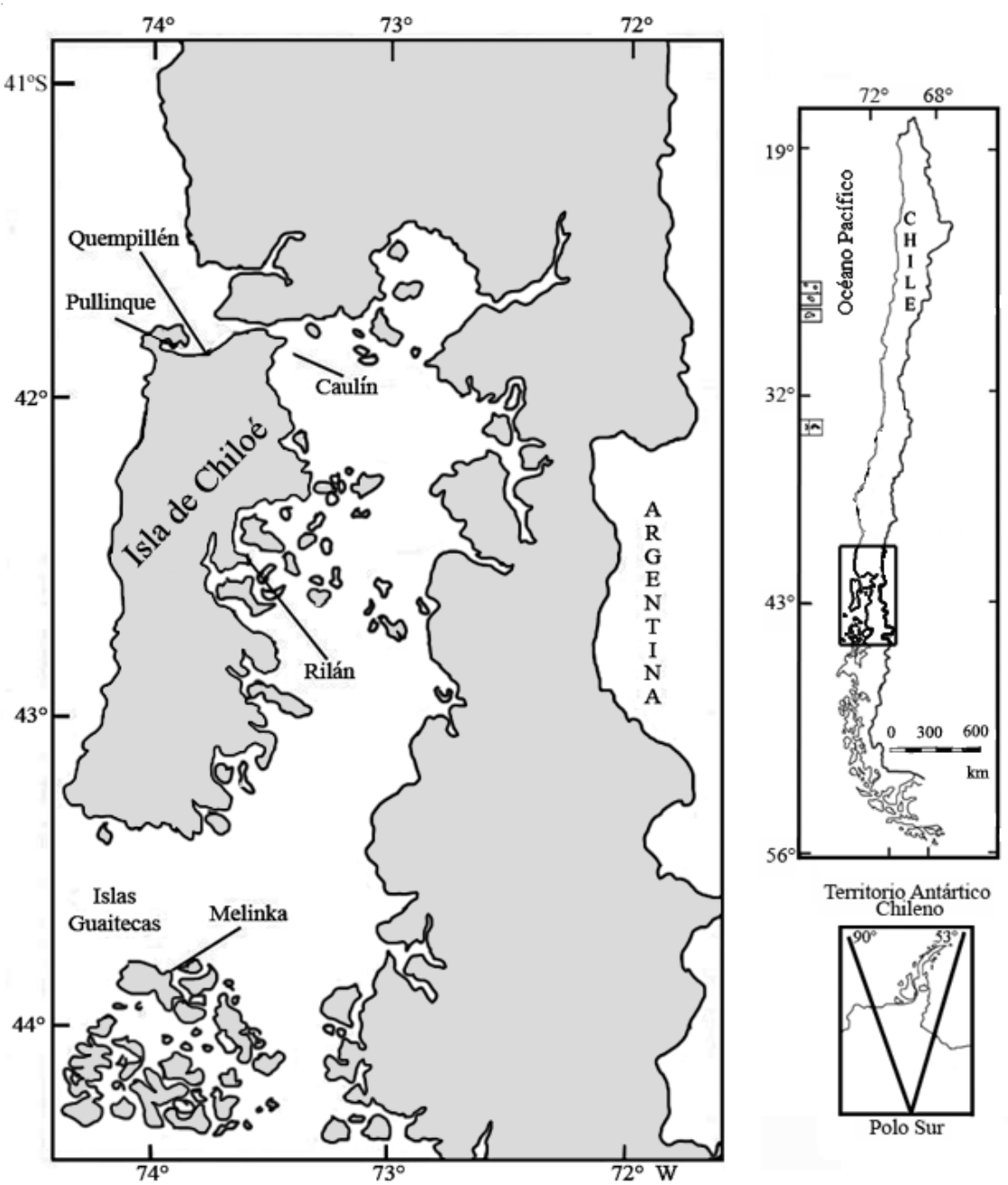

Figura 1

Localidades de muestreo de poblaciones de Ostrea chilensis para análisis genéticos con marcadores RAPds (Quempillén, Pullinque, Caulín, Rilán y Melinka)

Sampling sites of Ostrea chilensis populations for genetic analyses with RAPDs markers (Quempillén, Pullinque, Caulín, Rilán and Melinka)

\section{Extracción deADN}

Una muestra de tejido (50-100 mg) se maceró y digirió en una solución de $500 \mu \mathrm{L}$ de buffer lisis (EDTA $10 \mathrm{mM}$, Tris-HCL $10 \mathrm{mMpH} 8,5, \mathrm{NaCL} 20 \mathrm{mM}), 50 \mu \mathrm{L}$ de SDS al $20 \%$ p/v y $5 \mu \mathrm{L}$ de proteinasa $\mathrm{K}$ e incubó a $37^{\circ} \mathrm{C}$ durante $12 \mathrm{~h}$ (Toro 1998). Luego se adicionaron a la reacción $500 \mu \mathrm{L}$ de una solución de fenol-cloroformo-isoamil alcohol en proporciones de 25:24:1 respectivamente (Sigma) y se centrifugó a $13.000 \mathrm{G}$ durante $15 \mathrm{~min}$ a $10^{\circ} \mathrm{C}$. El sobrenadante resultante se resuspendió en $800 \mu \mathrm{L}$ de etanol al 95\% mantenido previamente a $-20^{\circ} \mathrm{C}$. Una vez precipitado el $\mathrm{ADN}$, las muestras se centrifugaron a $6.500 \mathrm{G}$ durante $3 \mathrm{~min}$ a $10^{\circ} \mathrm{C}$, resuspendiendo el pellet obtenido en $200 \mu \mathrm{L}$ de agua ultradestilada (Toro et al. 2004). La cantidad y calidad de ADN obtenido fue visualizado por medio de electroforesis en gel de agarosa $(1,5 \%)$ con TBE y bromuro de etidio. La calidad e integridad del ADN se comparó con un ladder de 2300 pb. La cuantificación y pureza del ADN se realizó por espectrofotometría (González 2006). 
Vol. 44, N², 2009

\section{Amplificación y electroforesis}

Se utilizaron cuatro partidores (Tabla 1) disponibles comercialmente (Biosonda SA) para llevar a cabo el análisis de variabilidad genética. Estos fueron elegidos en base al número e intensidad de las bandas, reproducibilidad, grado de polimorfismo, visualización y repetibilidad de las bandas. Para la reacción de amplificación se utilizó un volumen de mezcla total de $25 \mu \mathrm{L}$, consistente en $1 \mu \mathrm{L}$ de $\mathrm{ADN}$ genómico (50 ng $\left.\mu \mathrm{L}^{-1}\right), 7,5 \mu \mathrm{L}$ de dNTPs $(2 \mathrm{mM}), 1,75 \mu \mathrm{L}$ de $\mathrm{MgCl}_{2}$ (25 mM), 2,5 $\mu \mathrm{L}$ PCR-buffer 10x, $3 \mu \mathrm{L}$ del partidor (10 $\left.\mu \mathrm{M} \mu \mathrm{L}^{-1}\right), 1$ unidad de Taq ADN polimerasa $\left(5 \mu \mathrm{L}^{-1}\right)$ (Invitrogen) y $9,05 \mu \mathrm{L}$ de agua ultradestilada. La amplificación se llevó a cabo en un Termociclador Amplitron ${ }^{\circledR}$ II (Thermolyne) y el programa utilizado contó con un ciclo de desnaturalización del $\mathrm{ADN}$ a $95^{\circ} \mathrm{C}$ por $15 \mathrm{~s}$, seguido de 36 ciclos a $94^{\circ} \mathrm{C}$ por $30 \mathrm{~s}$, un ciclo de hibridación a $33^{\circ} \mathrm{C}$ por $30 \mathrm{~s}$ y una fase de extensión a $72^{\circ} \mathrm{C}$ por $1 \mathrm{~min}$. $\mathrm{Al}$ término de los 36 ciclos el programa ejecutó una extensión final de 2 min a $72^{\circ} \mathrm{C}$ y finalmente el producto amplificado fue conservado a $4^{\circ} \mathrm{C}$.

Los fragmentos de ADN amplificados se separaron por medio de electroforesis en gel de agarosa $(1,5 \%)$ sumergido en buffer TBE 0,5 X (44,5 mM tris base, 44,5 mM ácido bórico, $1 \mathrm{M}$ EDTA, pH 8) con bromuro de etidio (10 mg $\mathrm{mL}^{-1}$ ) (Toro et al. 2002, 2004). Se incluyó un patrón estándar (Ladder 100 bp, Invitrogen), el cual permitió discriminar los productos amplificados de acuerdo a su peso molecular. Las bandas en los geles fueron visualizadas posteriormente en un transiluminador UV y fotografiadas con una cámara digital.

\section{Análisis de datos}

Los patrones de bandas obtenidos (RAPDs) se analizaron visualmente para cada muestra, en base a presencia (1) o ausencia (0) de estas bandas para cada uno de los cuatro partidores utilizados (Toro et al. 2004). Los análisis genéticoestadísticos se llevaron a cabo para 30 individuos de cada población (aquellos individuos donde se obtuvo la mejor calidad de ADN de cada una de las poblaciones), utilizando el programa computacional POPGENE versión 2.6.2 (Population Genetics Software) (Yeh \& Boyle 1997). Para los análisis se incluyó el coeficiente de endogamia Fis = 0,109 (Toro \& Aguila 1996). En dicho estudio el valor de Fis no fue estadísticamente significativo, por lo tanto, en los análisis llevados a cabo en este estudio se asumió esta misma condición de equilibrio. Se determinó en cada población el número observado de alelos, número efectivo de alelos (Kimura \& Crow 1964) y diversidad genética (Nei 1973). Además, se determinó la frecuencia génica general, flujo génico (McDermott \& McDonald 1993) y el análisis de diversidad genética en poblaciones subdivididas (Nei
Tabla 1

Secuencia de partidores RAPD utilizados en Ostrea chilensis Sequence of RAPD primers used in Ostrea chilensis

\begin{tabular}{ccc}
\hline Partidor & Secuencia 5, $\rightarrow$ 3, & $\begin{array}{c}\mathrm{N}^{\circ} \text { total de bandas } \\
\text { marcadas }\end{array}$ \\
\hline OST1 & GGGCGCGAGT & 10 \\
OST2 & GCGGCTGGAG & 12 \\
OST3 & TAGCCCGCTT & 10 \\
OST4 & AACACACGAG & 11 \\
\hline
\end{tabular}

1987). Se estimó la identidad y distancia genética (Nei 1972) y se construyó un dendrograma de identidad genética entre poblaciones (Yeh \& Boyle 1997). Se llevó a cabo una prueba de Mantel, la cual permitió establecer el grado de correlación entre matrices de comparaciones pareadas de datos utilizando 5000 permutaciones al azar. A partir de esta prueba se obtuvo una correlación entre la distancia genética $\mathrm{D}$ (Nei 1972) y la distancia geográfica (costera lineal en km). Este análisis se llevó a cabo con el programa computacional PopTools versión 2.6.2 (Hood 2004).

\section{Resultados}

El número de bandas (RAPDs) generadas por los cuatro partidores analizados varió entre 10 y 12 obteniendo un total de 43 bandas reproducibles (Tabla 1), las cuales presentaron un alto grado de polimorfismo (Figs. 2 y 3). A partir de la frecuencia de alelos RAPD calculada para cada locus se obtuvo los parámetros de diversidad genética intra e interpoblacional correspondientes a las cinco localidades muestreadas.

El valor promedio interpoblacional de diversidad genética de Nei (1973) fue de 0,2846 $\pm 0,1514$, (28\%). Los valores de diversidad génica intrapoblacional correspondieron a un 16,9\% (Pullinque), 20,3\% (Rilán), 22,7\% (Caulín), 23,5\% (Melinka) y un 24,6\% (Quempillén). El resumen de la diversidad genética intrapoblacional e interpoblacional para los 43 loci en las cinco poblaciones de Ostrea chilensis se presenta en la Tabla 2. Los mayores porcentajes de loci polimórficos correspondieron a Caulín y Melinka con 72,09\%, seguidos de Quempillén con 67,44\% y Quetalmahue, Rilán con 55,81\%.

El valor promedio del flujo génico obtenido con los 43 loci RAPD tuvo valor de $\mathrm{Nm}=1,587$ (Tabla 2). Las distancias genéticas observadas entre los pares de poblaciones estudiadas de Ostrea chilensis, no presentaron valores significativos de diferenciación poblacional, con un 
Tabla 2

Parámetros de diversidad genética en cinco poblaciones de Ostrea chilensis

Genetic diversity parameters in five populations of Ostrea chilensis

\begin{tabular}{|c|c|c|c|c|c|c|}
\hline & Caulín & Melinka & Pullinque & Quempillén & Rilán & Interpoblacional \\
\hline Diversidad génica de Nei (h) & 0,2270 & 0,2359 & 0,1697 & 0,2465 & 0,2031 & 0,2846 \\
\hline Desviación estándar & 0,1878 & 0,2012 & 0,1884 & 0,2083 & 0,2007 & 0,1514 \\
\hline Índice de información de Shannon (I) & 0,3465 & 0,3540 & 0,2603 & 0,3644 & 0,3035 & 0,4421 \\
\hline Desviación estándar & 0,2649 & 0,2806 & 0,2722 & 0,2912 & 0,2892 & 0,1898 \\
\hline Número de loci polimorficos & 31 & 31 & 24 & 29 & 24 & 43 \\
\hline$\%$ de loci polimorficos & $72,09 \%$ & $72,09 \%$ & $55,81 \%$ & $67,44 \%$ & $55,81 \%$ & $100 \%$ \\
\hline Coeficiente de diferenciación génica (Gst) & & & & & & 0,2395 \\
\hline Flujo génico (Nm) & & & & & & 1,5877 \\
\hline
\end{tabular}

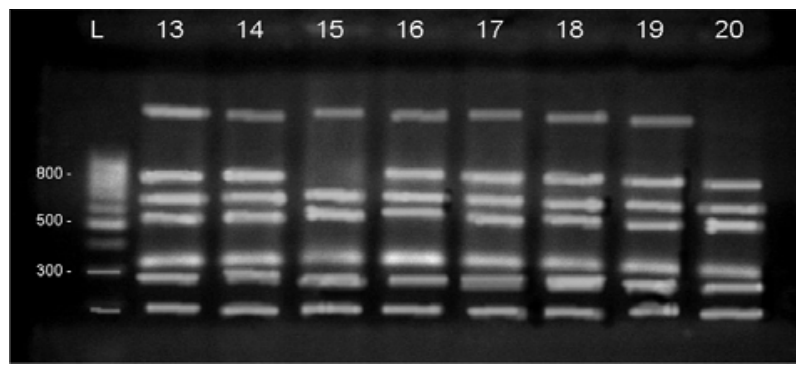

Figura 2

Ostrea chilensis. Patrones de banda de 8 individuos (13-20) pertenecientes a la población natural de Pullinque generados con el partidor OST3. (gel de agarosa al 1,5\%, concentración de $A D N=500 \mathrm{ng} \mu \mathrm{L}^{-1}, L=$ escala de 100 bp)

Ostrea chilensis. Banding patterns from 8 individuals (13-20) belonging to the Pullinque natural population, generated by primer OST3. (1.5\% agarose gel, DNA concentration $=500 \mathrm{ng} \mu \mathrm{L}^{-1}, \mathrm{~L}=100$ bp ladder)

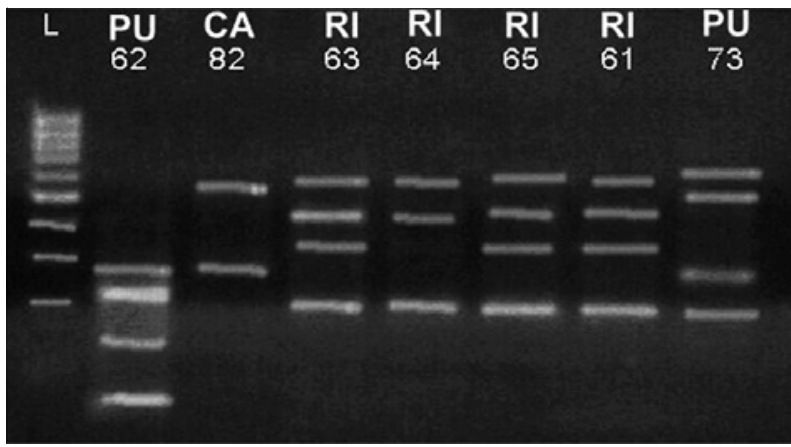

Figura 3

Ostrea chilensis. Comparación de patrones de banda generados por los partidores OST1 (para ostras 62 y 65), OST2 (para ostras 82 y 61), OST3 (para ostras 63 y 73) y OST4 (para ostra 64) en individuos pertenecientes a las poblaciones de Pullinque (PU),

Caulín (CA) y Rilán (RI). (gel de agarosa al 1,5\%, concentración de ADN $=500 \mathrm{ng} \mu \mathrm{L}^{-1}, \mathrm{~L}=$ escala de 100 bp)

Ostrea chilensis. Comparison of banding patterns generated by primers OST1 (for oyster 63 and 65), OST2 (for oysters 82 and 61 ), OST3 (for oysters 63 and 73) and OST4 (for oyster 64) for individuals belonging to the populations of Pullinque (PU), Caulín (CA) and Rilán (RI). (1.5\% agarose gel, DNA concentration $=500 \mathrm{ng} \mu \mathrm{L}^{-1}, \mathrm{~L}=100 \mathrm{bp}$ ladder) 
Tabla 3

Correlación entre las distancias geográficas $(\mathbf{k m})$ y las distancias genéticas D de Nei (en
paréntesis), entre cinco poblaciones de Ostrea chilensis

Correlation between geographic distances $(\mathrm{km})$ and Nei's unbiased genetic distance D (in parentheses) separating five populations of Ostrea chilensis

\begin{tabular}{lcccc}
\hline \multicolumn{1}{c}{ Población } & Caulín & Quempillén & Pullinque & Rilán \\
\hline Caulín & --- & & & \\
Quempillén & $20,5(0,0713)$ & --- & & \\
Pullinque & $34,6(0,1007)$ & $15,3(0,1329)$ & --- & -- \\
Rilán & $82,3(0,0843)$ & $73,4(0,0828)$ & $80,6(0,1163)$ & $145(0,1549)$ \\
Melinka & $226(0,1231)$ & $215(0,1296)$ & $220(0,1543)$ & --- \\
\hline
\end{tabular}

$\mathrm{r}=0,605(P<0,05)$

promedio de 0,113. Los valores de distancia genética (D) de Nei (1972), fluctuaron entre 0,0713 (Caulín-Quempillén) y 0,1549 (Rilán-Melinka) (Tabla 3). Se observó una moderada separación de la población Melinka, con respecto a las poblaciones localizadas en la isla de Chiloé. Sin embargo, los valores obtenidos para la distancia genética entre poblaciones no son significativos e indican que no existe una subdivisión genético-poblacional entre las cinco localidades estudiadas. El análisis de valores de identidad (I), graficado mediante el sistema UPGMA, se ilustran en la Fig. 3.

La prueba de Mantel utilizando 5000 permutaciones produjo un coeficiente de correlación entre la distancia genética y geográfica de $r=0,605(P<0,05)$ (Tabla 3$)$.

\section{Discusión}

Las características de historia de vida de Ostrea chilensis la convierten en una especie única dentro de la familia Ostreidae y es por esta razón que varios estudios biológicos se han focalizado en esta especie (Solís \& Eberhard 1979, Chanley \& Dinamani 1980, Guiñez 1988, Toro \& Chaparro 1990, Toro 1995, Toro et al. 1995, Toro \& Aguila 1996, Toro et al. 2004). Sin embargo, el presente estudio es el primero en utilizar marcadores moleculares basados en el análisis de $\mathrm{ADN}$, con el fin de determinar la actual estructura poblacional e intercambio genético entre las poblaciones naturales dentro de la reducida zona de distribución natural de la ostra chilena.

La diversidad genética interpoblacional obtenida para Ostrea chilensis en este estudio $(0,2846)$ está por sobre el rango estimado para invertebrados marinos $(0,122 ; 0,145 \mathrm{y}$
0,230 según Ward et al. (1992), Fujio et al. (1983), Hermosilla (2004), respectivamente). Así también, el polimorfismo genético, es decir, las diferentes formas en que un gen puede expresarse en una población, alcanzó altos niveles (55,8\% a $72,0 \%)$, comparado con la especie Ostrea edulis (18,2\% y $40,9 \%$ ), cuyo polimorfismo fue medido mediante la técnica de electroforesis enzimática (Saavedra et al. 1993). En otro estudio en la especie O. edulis, Beaumont et al. (2006) utilizando marcadores microsatelitales obtuvieron valores promedio para el número de alelos por locus que variaron entre 10,0 y 13,5, lo que se debería, según los autores, al bajo tamaño muestreal que fluctuó en promedio entre 9,3 y 33,5 individuos por localidad. Es necesario enfatizar que la diferencia en la estrategia de dispersión larval entre $O$. chilensis y $O$. edulis puede causar una estructura genéticopoblacional distinta (Hunt 1993, Hoskin 1997), puesto que esta última posee un período de vida larval pelágica que abarca aproximadamente unos 10 a 12 días (Walne 1974), mientras que en la ostra chilena este período es de solo unos minutos a horas (DiSalvo \& Martínez 1985).

En la mayoría de los moluscos bivalvos existe una abundante variación espacial tanto en morfología (Innes \& Bates 1999, Toro et al. 2008) como en frecuencia génica (Fujio et al. 1983, Seed \& Suchanek 1992, Bates \& Innes 1995, Comesaña et al. 1998). Esta variación es el resultado de un balance de fuerzas selectivas que tienden a producir diferenciación genética local (mutación y deriva génica) y otras que tienden a producir homogeneidad genética (flujo génico como movimiento de gametos, individuos e inclusive la población total) (Slatkin 1987). Si dos poblaciones se encuentran completamente diferenciadas en sus frecuencias alélicas, significa que el flujo génico entre ellas ha sido 
reducido durante un largo intervalo de tiempo, producto de distancias o barreras geográficas o procesos oceanográficos. Por otra parte, cuando dos poblaciones son muy similares en sus frecuencias alélicas, se interpreta como si ambas poblaciones conforman una misma unidad reproductiva o que su separación es muy reciente; por lo tanto no les ha permitido acumular diferencias genéticas que permitan identificarlas como dos poblaciones separadas. (Spanakis et al. 1989). El flujo génico interpoblacional observado para O. chilensis $(\mathrm{Nm}=1,5877)$ indica que el flujo génico estaría actuando por sobre la deriva génica, impidiendo con esto la diferenciación genética en las poblaciones (Slatkin 1987). Este resultado se contrapone con lo propuesto por Toro \& Chaparro (1990), quienes de acuerdo a las características de la historia de vida de $O$. chilensis relacionadas a la fase larval pelágica de tan solo algunos minutos, sugieren un gran potencial para la diferenciación genética de esta especie. Algunas de las explicaciones para el valor de flujo génico $(>1)$ obtenido en el presente estudio, pudiesen ser consecuencia del movimiento antropogénico de juveniles (semillas) para el repoblamiento y cultivo desde los centros productores de semillas naturales (Pullinque y Quempillén) hacia las ostriculturas (Solis \& Eberhard 1979, Toro \& Chaparro 1990, Toro \& Águila 1996) y a procesos oceanográficos tales como corrientes superficiales (Silva et al. 1998) y/o al fenómeno de balseo ('rafting') (Thiel 2003). Por otro lado, los bajos valores de flujo genético entre Pullinque y Melinka pueden ser atribuidos a un factor oceanográfico costero localizado aproximadamente a los $43^{\circ} \mathrm{S}$ y que correspondería a la corriente de deriva del oeste (WWD) (Strub \& Mesías 1998), la cual actuaría como una barrera geográfica natural impidiendo una dispersión larval efectiva desde las localidades de Pullinque y Melinka. Esta hipótesis se basa en que la distancia geográfica no sería el principal factor determinante para los valores de flujo génico entre pares de poblaciones, ya que el flujo génico registrado entre Caulín y Melinka fue notoriamente mayor que entre Pullinque y Melinka cuya distancia geográfica lineal es menor.

Las distancias genéticas observadas entre los pares de poblaciones estudiadas de Ostrea chilensis no presentaron valores significativos de diferenciación poblacional, con un promedio de 0,113; el rango comprendido por estas distancias genéticas fue de 0,0713 (Caulín-Quempillén) a 0,1549 (Rilán-Melinka). Hassanien et al. (2004) establecen que los valores de distancia genética de Nei (1972) para poblaciones conespecíficas se presentan en un valor promedio cercano a $0,05(0,002-0,07)$, para especies congenéricas un promedio de $0,30(0,03-0,61)$, y para géneros confamiliares un promedio de $0,90(0,58-1,21)$. En base a estos valores, el grado de diferenciación genética de
O. chilensis se encontraría entre el nivel de especies conespecíficas y congenéricas.

El dendrograma (UPGMA) que graficó las diferencias genéticas entre poblaciones basadas en los valores de Identidad genética (I), separó las poblaciones de Ostrea chilensis en dos grupos; uno compuesto por las poblaciones de la Isla de Chiloé y otro grupo conformado por la población perteneciente a las Islas Guaitecas (Melinka). Esta separación podría ser producto más que de la distancia geográfica a la presencia de corrientes como la WWD (Strub \& Mesías 1998) y corrientes superficiales en el Golfo de Corcovado (Silva et al. 1998) que pueden estar actuando como barreras geográficas.

Toro \& Aguila (1996) realizaron un estudio con aloenzimas en las poblaciones de Ostrea chilensis, evidenciando baja variabilidad genética intrapoblacional y relativamente alta diversidad genética interpoblacional. Además, describen que $O$. chilensis no representa una simple población genéticamente homogénea, sino mas bien las poblaciones del norte de la Isla de Chiloé corresponderían a poblaciones reproductivamente aisladas de las poblaciones del sector sur de la Isla (Toro \& Aguila 1996). El presente estudio es coincidente con el anterior ya que diferencia genéticamente a la población de Melinka con las poblaciones pertenecientes a la Isla de Chiloé. Sin embargo, Toro \& Aguila (1996) no estimaron el flujo génico entre poblaciones y lamentablemente tampoco existen en la literatura valores de este parámetro para $O$. chilensis que permitan hacer comparaciones.

Desde el punto de vista genético, el concepto de 'stock' se define como una población panmíctica de individuos emparentados y genéticamente distintos de otras poblaciones de la misma especie (Shaklee et al. 1990). Sin embargo, la extensa distribución geográfica y la presencia de barreras que restringen el libre movimiento de los individuos sugiere que no existiría una panmixia total entre los individuos de una misma especie, lo que da como resultado que una especie esté formada por numerosas poblaciones panmícticas o demos (Galleguillos et al. 1997). En algunos invertebrados marinos, la baja dispersión larval y poca movilidad de los adultos tienen como consecuencia reducidos tamaños en los stocks, lo cual les hace más vulnerables a los efectos de la sobrepesca. Además, estudios genéticos indican que existe una alta incidencia de especiación críptica en invertebrados marinos, lo que añade una mayor dificultad para la identificación de stocks (Thorpe et al. 2000, Jolly et al. 2005). Por consiguiente, y considerando los resultados obtenidos en este estudio, no es posible identificar una clara conformación de stocks en las poblaciones estudiadas de O. chilensis, debido a que no existe una línea base y, por ende, los resultados obtenidos sólo pueden ser considerados 
como preliminares a la hora de desarrollar políticas y programas de manejo adecuados para la ostra chilena. Es importante llevar a cabo más estudios de este tipo, que permitan esclarecer la conformación de unidades reproductivas de esta especie dentro de su zona de distribución natural. Por el momento, y de acuerdo a este estudio, Melinka sería la única población que podría ser considerada como un stock discreto, ya que fue la población genéticamente más distinta y geográficamente más distante.

La prueba de Mantel reveló una correlación significativa entre las distancias genéticas y geográficas $(P<0,05)$. Lo anterior indicaría que la distancias genéticas registradas entre las poblaciones de Ostrea chilensis, mediante marcadores moleculares RAPD, dependen en algún grado de las distancias geográficas existentes entre las localidades muestreadas lo que resulta esperable para esta especie dada su estrategia reproductiva, especialmente al incluir en el análisis la población de Melinka, que se encuentra geográficamente mas distante. Es probable que al realizar la prueba de Mantel utilizando solo la distribución natural de poblaciones de $O$. chilensis dentro de la Isla de Chiloé (con reducidas distancias geográficas), pudiese afectar en alguna medida la sensibilidad del análisis y con ello el grado de correlación entre estas variables, considerando, además, la existencia de traslados antropogénico de semillas ya sea para fines de repoblamiento o comercial (acuicultura) de esta especie.

Este estudio, cuyo objetivo consistió en evaluar niveles de variación genética entre poblaciones naturales de Ostrea chilensis, debe tomarse como un punto de partida para realizar futuros monitoreos genéticos dentro de estas mismas localidades, con el fin de controlar el impacto de los individuos cultivados sobre la diversidad de las poblaciones naturales, para ir evaluando asertivamente los programas de manejo que deben aplicarse a esta especie.

\section{Agradecimientos}

Los autores agradecen los comentarios de dos evaluadores anónimos que permitieron mejorar sustantivamente el manuscrito. Este estudio fue financiado por los proyectos FONDECYT 1050341 y 1095061.

\section{Literatura citada}

Aagaard JEK, V Krutivskii \& SH Strauss. 1998. RAPDs and allozymes exhibit similar levels of diversity and differentiation among populations and races of Douglas-fir. Heredity 81: 6978.

Bardakci F \& DOF Skibinski. 1994. Applications of the RAPD technique in tilapia fish: species and subspecies identification. Heredity 73: 117-123.
Bates JA \& DJ Innes. 1995. Genetic variation among populations of Mytilus spp. in eastern Newfoudland. Marine Biology 124: 417-424.

Beaumont A, M Truebano-Garcia, S Höng \& P Low. 2006. Genetics of Scottish populations of the native oyster, Ostrea edulis: gene flow, human intervention and conservation. Aquatic Living Resources 19: 389-402.

Bustos E, R Guiñez, E Olavarría, A Paredes \& J Valencia. 1991. Desarrollo de técnicas de producción de semillas y repoblación de recursos bentónicos. Programa de las Naciones Unidas para el Desarrollo, 23 pp. Informe Técnico. Instituto de Fomento Pesquero, Valparaíso.

Caetano-Anolles G, BJ Bassam \& PM Gresshoff. 1991a. DNA Amplifications Fingerprinting using very short arbitrary oligonucleotide primers. Bio/Technology 9: 553-557.

Caetano-Anolles G, BJ Bassam \& PM Gresshoff. 1991b. DNA Amplifications Fingerprinting: A strategy for genome analysis. Plant Molecular Biology Reporter 9: 294-307.

Callejas C \& MD Ochando. 2002. Phylogenetic relatioship among spanish barbus species (Pisces, Cyprinidae) shown by RAPD markers. Heredity 89: 36-43.

Chanley P \& P Dinamani. 1980. Comparative descriptions of some oyster larvae from New Zealand and Chile, and a description of a new genus of oyster Tiostrea. New Zealand Marine and Freshwater Research 14: 103-120.

Comesaña AS, JE Toro, DJ Innes \& RJ Thompson. 1998. A molecular approach to the ecology of a mussel (Mytilus edulisM. trossulus) hybrid zone on the east coast of Newfoundland, Canada. Marine Biology 133: 213-221.

Di Salvo LH \& E Martínez. 1985. Culture of Ostrea chilensis Philippi 1845 in a north central Chilean coastal bay. Biología Pesquera 14: 16-22.

Elo K, S Ivanoff, JA Vuorinen \& J Piironen. 1997. Inheritance of RAPD markers and detection of interespecific hybridization with brown trout and Atlantic salmon. Aquaculture 152: 5565.

Fujio Y, R Yamanaka \& PT Smith. 1983. Genetic variation in marine molluscs. Bulletin of the Japanese Society of Scientific Fisheries 49: 1809-1817.

Galleguillos R, L Troncoso, J Monsalves \& C Oyarzún. 1997. Diferenciación poblacional en la sardina chilena Strangomera bentincki (Pisces: Clupeidae) análisis genético de variabilidad proteínica. Revista Chilena de Historia Natural 70: 351-361.

González CP. 2006. Estructura genético-poblacional y flujo génico en Ostrea chilensis (Philippi 1845) en un amplio rango de distribución utilizando marcadores moleculares RAPDs. Tesis de Biología Marina, Facultad de Ciencias, Universidad Austral de Chile, Valdivia, 69 pp.

Guiñez R. 1988. Mejoramiento genético en recursos marinos: situación actual y perspectivas. Investigación Pesquera 35: 113121.

Hassanien HA, M EInady, A Obeida \& H Itriby. 2004. Genetic diversity of Nile tilapia populations revealed by randomly amplified polymorphic DNA(RAPD). Aquaculture Research 35: 587-593. 
Hermosilla CA. 2004. Variación morfológica y genética entre poblaciones del pulpo de Chiloé Enteroctopus megalocyatus (Cephalopoda, Octopoda) en el sur de Chile y Argentina. Tesis de Biología Marina, Facultad de Ciencias, Universidad Austral de Chile, Valdivia, 96 pp.

Holms SP, R Witbaard, J Van der Meers. 2003. Phenotypic and genotypic population differentiation in the bivalve mollusc Arctica islandica: result from RAPD analysis. Marine Ecology Progress Series 254: 163-176.

Hood GM. 2004. PopTools. Version 2.6.2. [en línea] <http:// www.cse.csiro.au/poptools/>

Hoskin MG. 1997. Effects of contrasting modes of larval development on the genetics structure of populations of three species of prosobranch gastropods. Marine Biology 127: 647-656.

Hunt A. 1993. Effects of contrasting patterns of larval dispersal on the genetic connectedness of local populations of two intertidal starfish, Patiriella calcar and P. exigua. Marine Ecology Progress Series 92: 179-186.

Innes DJ \& JA Bates. 1999. Morphological variation of Mytilus edulis and Mytilus trossulus in eastern Newfoundland. Marine Biology 133: 691-699.

Jolly MT, D Jollivet, F Gentil, E Thiébaut \& F Viard. 2005. Sharp genetic break between Atlantic and English Channel populations of the polychaete Pectinaria koreni, along the north coast of France. Heredity 94: 23-32.

Keyvanshokooh S \& MR Kalbassi. 2006. Genetic variation of Rutilus rutilus caspicus (Jakowlew 1870) populations in Iran based on random amplified polymorphic DNA markers: a preliminary study. Aquaculture Reserach 37: 1437-1440.

Kimura M \& JF Crow. 1964. The number of alleles that can be maintained in a finite population. Genetics 49: 725-738.

Lépez I. 1983. El cultivo de Ostrea chilensis en la zona central y sur de Chile, Memorias de la Asociación Latinoamericana de Acuicultura 5: 117-127.

Liu ZJ \& JF Cordes. 2004. DNA marker technologies and their applications in aquaculture genetics. Aquaculture 238: 1-37.

Lynch M \& B Milligan. 1994. Analysis of population genetic structure with RAPD markers. Molecular Ecology 3: 91-99.

McDermott JE \& BA Mc Donald. 1993. Gen flow in plants pathosystems. Annual Review of Phytopathology 31: 353373.

Nei M. 1972. Genetic distance between populations. The American Naturalist 106: 283-292.

Nei M. 1973. Analysis of gene diversity in subdivided populations. Proceedings of the National Academy of Science of the United States of America 70: 3321-3323.

Nei M. 1987. Molecular evolutionary genetics, 132 pp. Columbia University Press, New York.

Nei M. 1998. Estimation of average heterozygosity and genetic distance from a small number of individuals. Genetics 89: 583590.

Patwary MU, EL Kenchington, CJ Bird \& E Zouros. 1994. The use the random amplified polymorphic DNA markers in genetic studies of the sea scallop Placopecten magellanicus (Gmelin, 1791). Journal of Shellfish Research 13: 547-553.

Patwary MU, RM Ball, CJ Bird, B Gjetvaj, S Sperker, E Kenchington \& E Zouros. 1995. Genetic markers in the sea scallop and their application to aquaculture. Bulletin of the Aquaculture Association of Canada 94: 18-20.

Pearson CVM, AD Rogers \& M Sheader. 2002. The genetic structure of the rare lagoonal sea anemone, Nematostella vectensis Stephenson (Cnidaria: Anthozoa) in the United Kingdom based on RAPD analysis. Molecular Ecology 11: 2285-2293.

Ramorino L. 1970. Estudios preliminares sobre la crianza de Ostrea chilensis en el laboratorio. Biología Pesquera 4: 1732.

Saavedra C, C Zapata, A Guerra \& G Álvarez. 1993. Allozyme variation in european populations of the oyster Ostra edulis. Marine Biology 115: 85-95.

Scheltema RS. 1989. Planktonic and non-planktonic development among prosobranch gastropods and its relationship to the geographic range of species. En: Ryland S \& PA Tyler (eds). Reproduction, genetics and distribution of marine organisms, pp. 183-188. Olsen \& Olsen, Fredensborg.

Seed R \& TH Suchanek. 1992. Population and community ecology of Mytilus. En: Gosling E (ed). The mussel Mytilus: ecology, physiology, genetics and culture, pp. 87-169. Elsevier, Amsterdam.

SERNAPESCA. 2004. Anuario estadístico pesquero 1993-2003. Servicio Nacional de Pesca, Valparaíso. [en línea] <http:// www.sernapesca.cl>

Shaklee JB, FW Allendorf, DC Morizot \& GS Whitt. 1990. Gene nomenclature for protein- coding loci in fish. Transactions of the American Fisheries Society 119: 2-15.

Silva PE \& CAM Russo. 2000. Techniques and statistical data analysis in molecular population genetics. Hydrobiologia 420: 119-135.

Silva N, C Calvete \& H Sievers. 1998. Masas de agua y circulación general para algunos canales australes entre Puerto Montt y Laguna San Rafael, Chile (Crucero CIMAR-FIORDO 1). Ciencia y Tecnología del Mar 21: 17-48.

Slatkin M. 1987. Gene flow and the geographic structure of natural populations. Science 236: 787-792.

Slatkin M \& NH Barton. 1989. A comparison of three indirect methods for estimating average levels of gene flow. Evolution 43: 1349-1368.

Solís I \& P Eberhard. 1979. Ostra. Ostrea chilensis Philippi Lamellibranchia Anisomyaria Ostreidae. En: Bahamonde N,A Sanhueza, C Martínez, O Rojas \& M Aguayo (eds). Estado actual de las principales pesquerías nacionales. Bases para un desarrollo pesquero. Moluscos, pp. 1-30. CORFO, Santiago

Spanakis E, N Tsimenides \& E Zouros. 1989. Genetic differences between populations of sardine, Sardina pilchardus, and anchovy, Engraulis encrasicolus, in the Aegean and Ionian seas. Journal of Fish Biology 35: 417-437.

Star B, S Apte \& JPA Gardner. 2003. Genetic estructuring among populations of the greenshell mussel Perna canaliculus 
revealed by analysis of randomly amplified polymorphic DNA. Marine Ecology Progress Series 249: 171-182.

Strub PT \& JM Mesías. 1998. Coastal oceanic circulation of western south America. Coastal ocean circulation off western South America. En: Robinson AR \& KH Brinks (eds), The Sea 11: 273-313. John Wiley, New York.

Thiel M. 2003. Rafting of benthic macrofauna: important factors determining the temporal succession of the assemblage on detached macroalgae. Hidrobiologia 503: 49-57.

Thorpe JP, AM Solé-Cava \& PC Watts. 2000. Exploited marine invertebrates: genetics and fisheries. Hydrobiologia 420: 165184.

Toro JE. 1995. A bibliography of literature on the Chilean oyster Ostrea chilensis (Philippi, 1845. Journal of Shellfish Research 14: 93-96.

Toro JE. 1998. PCR-based nuclear and mt DNA markers and shell morphology as an approach to study the taxonomy status of the Chilean blue mussel, Mytilus chilensis (Bivalvia). Aquatic Living Resources 11: 347-353.

Toro JE, AC Alcapán \& RA Stead. 2008. Cruzamientos interpoblacionales en Mytilus chilensis, un bivalvo de importancia comercial y sus efectos sobre el crecimiento en longitud de la valva durante la etapa larval. Archivos de Medicina Veterinaria 40: 299-303.

Toro JE \& PRAguila. 1996. Genetic differentiation of populations of the oyster Ostrea chilensis in southern Chile. Aquatic Living Resources 9: 75-78.

Toro JE \& OR Chaparro. 1990. Conocimiento biológico de Ostrea chilensis Philippi 1845. Impacto y perspectivas en el desarrollo de la ostricultura en Chile. En: Hernández A (ed). Cultivo de moluscos en América Latina, pp. 231-264. Centro Internacional de Investigaciones para el Desarrollo, Bogotá.

Toro JE \& PR Morandé. 1998. Effect of food ration and temperature on length of brooding period, larval development and size of pediveligers released in the Chilean oyster Ostrea chilensis. Journal of the World Aquaculture Society 29: 267270.

Toro JE, JA Ojeda \& AM Vergara. 2004. The genetic structure the Mytilus chilensis (Hupé, 1854) populations along the Chilean coast based on RAPDs analysis. Aquaculture Research 35: 1466-1471.

Toro, JE, RJ Thompson \& DJ Innes. 2002. Reproductive isolation and reproductive output in two sympatric mussel species (Mytilus edulis, M. trossulus) and their hybrids from Newfoundland. Marine Biology 141: 897-909.

Toro JE, MA Sanhueza, JE Winter, P Aguila \& AM Vergara. 1995. Selection response and heritability estimates for growth in the Chilean oyster Ostrea chilensis (Philippi, 1845). Journal of Shellfish Research 14: 87-92.

Walne PR. 1974. Culture of bivalve molluscs. 50 years experience at Conwy. 189 pp. Fishing News Books, Farnham, Surrey.

Ward RD, DOF Skibinski \& M Woodwork. 1992. Protein heterozygosity. Protein structure and taxonomic differentiation. Evolutionary Biology 26: 73-159.

Welsh J \& M McClellannd. 1990. Fingerprinting genomes using PCR with arbitrary primers. Nucleic Acids Research 18: 72137218.

Williams JGK, AR Kubelik, KJ Livak, JA Rafalski \& SV Tingey. 1990. DNA polymorphisms amplified by arbritrary primers are useful as genetic markers. Nuclear Acid Research 18: 6531-653.

Winter JE, JE Toro, JM Navarro, GS Valenzuela \& OR Chaparro. 1984. Recent developments, status and prospect of molluscan aquaculture on the Pacific coast of South American. Aquaculture 39: 95-134.

Yeh FC \& TJB Boyle. 1997. Population genetic analysis of codominant and dominant markers and quantitative traits. Belgium Journal of Botany 129: 157.

Recibido el 11 de noviembre de 2008 y aceptado el 29 de mayo de 2009 